\title{
Presence of Alternaria in Scalp of Patients with Alopecia Areata: Triggering Factor or Coexistence?
}

\author{
Hisham Diab Gaber ${ }^{1}$, Ahmed M. Mohamed ${ }^{2}$ and Reham M. Abdel Gaber ${ }^{1}$ \\ 1. Department of Dermatology, Venereology and Andrology, Faculty of Medicine, Assiut University, Assiut 71526, Egypt \\ 2. Department of Botany and Microbiology, Faculty of Science, Assiut University, Assiut 71526, Egypt
}

Received: July 27, 2015 / Accepted: July 30, 2015 / Published: July 30, 2015.

\begin{abstract}
AA (Alopecia areata) is the most frequent cause of inflammation-induced hair loss, affecting 0.1 to $0.2 \%$ of population worldwide. The development of organ-specific autoimmune reactions directed against anagen hair follicles seems to play a key role in the pathogenesis of alopecia areata. However, the triggering antigen(s) responsible for inducing autoimmune phenomena in these individuals remain unknown. Viral, bacterial or fungal pathogens have been implied as possible triggering factors of autoimmune reactions. The present study aims to identify the role of dematiaceous fungi in the pathogenesis of alopecia areata. 30 patients diagnosed clinically as alopecia areata and 30 normal age matched persons have undergone mycological examination. Mycology examination of the epidermal scrapings was done by DME (direct microscopic examination), culture on SDA (sabouraud's dextrose agar) and imaging. There is significantly higher percentage of positive results for Alternaria species by culture on sabouraud's agar in patients group (20\%) compared to controls $(13.3 \%) P$-value $<0.05$. The possible role of Alternaria antigens (e.g. antigens involved in melanin synthesis) in triggering autoimmunity in alopecia areata still needs further research on a wider scale of cases.
\end{abstract}

Key words: Alternaria, alopeciea areata.

\section{Introduction}

AA (Alopecia areata) is the most frequent cause of inflammation-induced hair loss, affecting 0.1 to $0.2 \%$ of population worldwide [1]. Despite its prevalence, there is little known about the underlying etiology and pathogenesis.

Several data indicate a role of genetic susceptibility to develop the disease [2]. Multiple candidate genes have been suggested [3].

The development of organ-specific autoimmune reactions directed against anagen hair follicles seems to play a key role in the pathogenesis of alopecia areata [4]. However, the triggering antigen(s) responsible for inducing autoimmune phenomena in these individuals remain unknown [3].

It has been suggested that hair follicle melanocytes,

Corresponding author: Hisham Diab Gaber, research fields: Dermatology, Venereology and Andrology. E-mail: hishamdiabg@hotmail.com. dermal papilla cells, or keratinocytes may contain the triggering antigens [5]. Viral, bacterial or fungal pathogens have been implied as possible triggering factors of autoimmune reactions [6]. Little is known about the pathogenic mechanisms by which these fungi cause disease, particularly in immunocompetent individuals [7].

The interest to study this point aroused from the case reported by Rudnicka and Lukomska [8] who described multiple hairless patches of focal hair loss with typical clinical and trichoscopy features of alopecia areata in a 13-year-old boy. Mycological examination of the scalp hair and epidermal scrapings reveled massive growth of Alternaria.

\section{Methodology}

Thirty patients diagnosed clinically as alopecia areata and 30 normal age matched persons have undergone mycological examination. 
Mycology examination of the epidermal scrapings from the scalp was done as follow:

(a) DME (Direct Microscopic Examination)

Scalp scrapings were examined under the high magnification lens of binocular microscope after treatment with $10 \% \mathrm{KOH}$ and lactophenol cotton blue stain. Positive sample is noted by the presence of fungal hyphae or budding cells [9].

(b) SDA (Culture on Sabouraud's Dextrose Agar)

Scalp scrapings were individually cultured on SDA which contained (g/L); Peptone 10, dextrose 20, agar 15 and Chloramphenicol 0.5. Cultures were prepared in plastic Petri plates and incubated at $28{ }^{\circ} \mathrm{C}$ for one to two weeks [9]. Positive cultures were examined and identified phenotypically according to de Hoog et al. [10].

(c) Imaging

Axiostar Trinocular microscope provided with Canon digital camera was used for taking photos of fungi observed on DME and after culturing (Figs. 1 and 2). Magnification rate of images was always $1,000 \mathrm{X}$.

\section{Results}

In patients group $36.7 \%$ (n:11) showed positive results by DME while only $20 \%$ (n:6) showed positive results for Alternaria by culture on sabourauds agar.

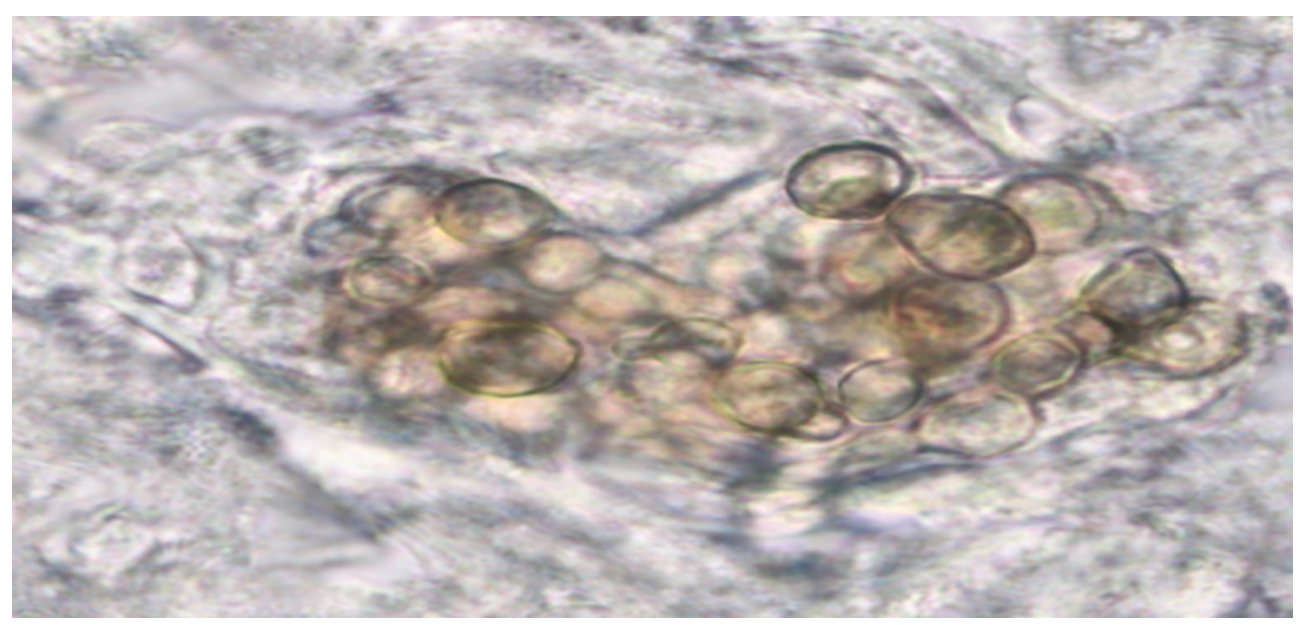

Fig. 1 Dark fungal cells in scalp scrapings by direct microscopic examination.

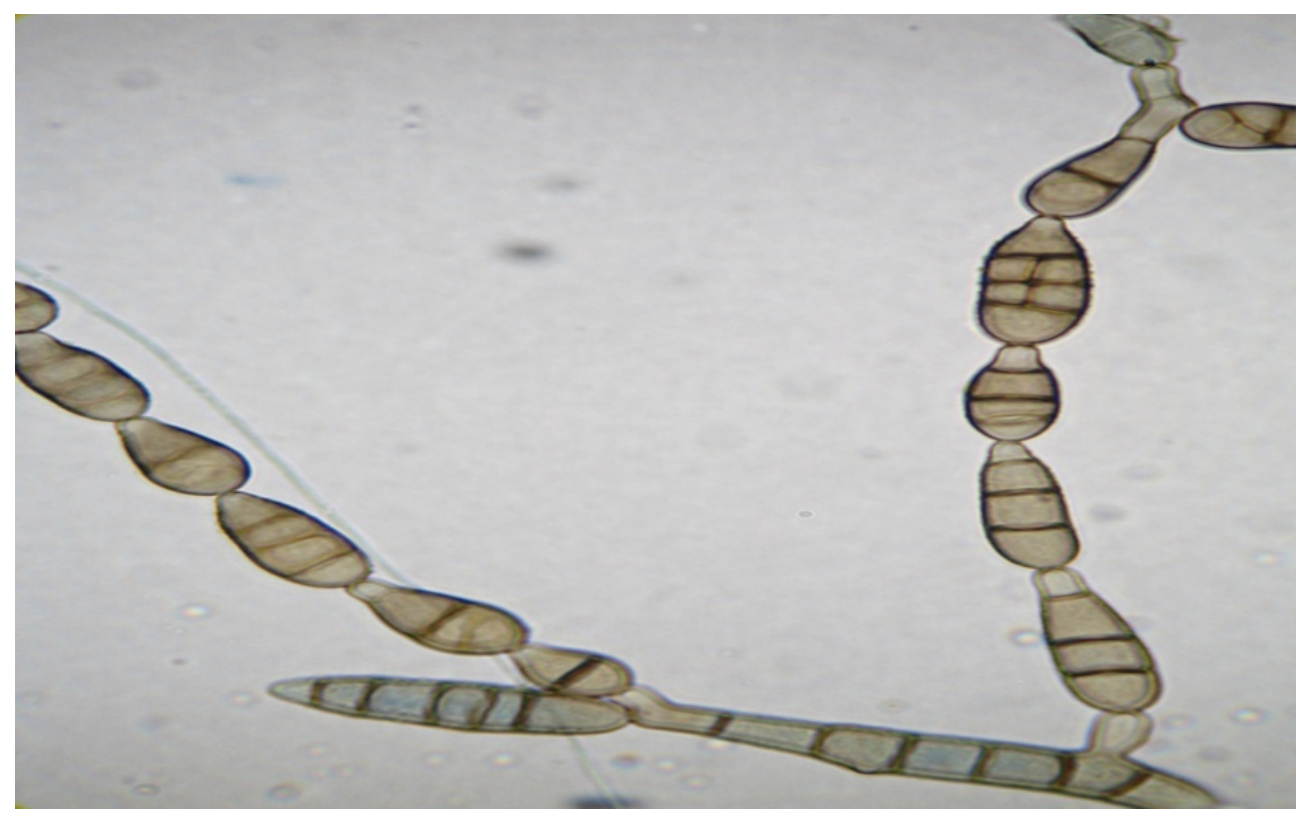

Fig. 2 Culture produced Alternaria alternate; muriform beaked dark conidia produced in long branch. 
Table 1 Comparison between alopecia areata group and control group according to mycological examination (DME and culture).

\begin{tabular}{llll}
\hline Item & Results (with alopecia) & Control (without alopecia) & $P$-value \\
\hline DME positive & $11(36.7 \%)$ & $8(26.6 \%)$ & N.S \\
Culture on SBD Alternaria (positive) & $6(20 \%)$ & $4(13.3 \%)$ & $<0.05$ \\
Mycologically free & $19(63.3 \%)$ & $22(73.3 \%)$ & N.S \\
\hline
\end{tabular}

$X^{2}$ test $=$ Chi-square test; N.S $=$ non significant $(>0.05)$.

Among the control group, 26.7\% (n:8) showed positive results by DME, only 13\% (n:4) gave positive results for Alternaria by culture on sabourauds agar.

There is significantly higher percentage of positive results for Alternaria species by culture on sabouraud's agar in patients group (20\%) compared to controls $(13.3 \%) P$-value $<0.05$.

\section{Discussion}

The hair follicle area is an immunologically privileged tissue, which is sheltered from immune surveillance by autoreactive T cells [4]. Autoimmune reactions are supposed to induce alopecia areata where, viral, bacterial or fungal pathogens have been implied as possible triggering factors [6].

In the present study, the co-association of fungi in cases with alopecia areata showed significantly higher percentage of positive results (20\%) for Alternaria species by culture on sabouraud's agar $(P<0.05)$ compared to controls $(13.3 \%)$. This is in agreement with [11], who reported that Alternaria are keratinolytic, dematiaceous (melanin-producing) fungi that are only occasionally implicated in opportunistic human diseases, such as skin and nail infections [12].

This can be explained by the hypothesis that antigens associated with melanogenesis are suggested to be potential triggers of autoimmunity in AA [13]. Thus, melanin producing fungi including Alternaria species may have a potentiating role in autoimmune mechanism of AA [14].

Melanins are a large group of diverse substances, which share similar properties. It may be speculated that exposure of predisposed individuals to fungal substances involved in melanin biosynthesis may contribute to development autoimmune reactions directed against human peptides involved in biosynthesis of follicular melanin. This would be possible in the mechanism of molecular mimicry, by-stander activation or epitope spreading [15].

This hypothesis may be supported by studies, which have shown that melanin and enzymes involved in melanin biosynthesis (e.g. tyrosinase) are highly immunogenic [16]. Various kinds of melanins revealed immunomodulatory activity by regulating cytokine production by T-lymphocytes and monocytes, as well as fibroblasts and endothelial cells [15]. It was even reported that the edible mushrooms, as they are a rich source of melanin and tyrosinase, may induce immunity to these antigens in humans [16].

Also, Kobayashi et al. [17], documented that Alternaria activates dendritic cells and produces potent Th2 adjuvant activity.

It has been documented that active alopecia areata is associated with presence of perifollicular inflammatory infiltrates, including predominantly CD8+ $\mathrm{T}$ lymphocytes [18].

Most authors suggest that AA is a T-cell mediated disease with prominent up-regulation of Th1 cytokines and downregulation of Th2 cytokines [19]. It has also been suggested that immune responses are regulated by Th1 cytokines in alopecia universalis and by Th2 cytokines in the patchy form of AA [20].

Moreover, other mononuclear cells and eosinophils are present perifollicular in cases with alopecia areata. The role of eosinophils in the pathogenesis of alopecia areata remains unclear. It has been reported that in most patients with diffuse AA the onset of disease was observed in spring and summer, suggesting that diffuse AA may be triggered by an increase in seasonal allergens. Authors indicate that common scalp pruritus 
before the first hair shedding, increased serum IgE level, and prominent eosinophilic infiltration may confirm this hypothesis [18].

However, Rudnicka and Lukomska [8] found that it is not unusual to observe subtle growth of Alternaria species in cultures from human scalp hair or epidermal scraping cultures.

Also, Knutsen et al. [21] by analysis of outdoor air routinely show presence of Alternaria spores, showing that most people are exposed to these fungi, but only few develop disease.

Alternaria is a large genus of species. These are mainly saprophytic indoor and outdoor airborne fungi. These fungi have pathogenic capacities over a broad range of hosts. Most species are plant pathogens [7]. Alternaria species are keratinophilic and keratinolylic fungi, found commonly on hair shafts of domestic animals, such as cats, dogs or horses [22].

In conclusion, the possible role of Alternaria antigens (e.g. antigens involved in melanin synthesis) in triggering autoimmunity in alopecia areata still needs further research on a wider scale of cases.

More studies are recommended to confirm this role by testing the effect of anti fungal therapy in treatment of cases of AA.

\section{References}

[1] Alkhalifah, A. 2013. “Alopecia Areata Update.” Dermatol Clin. 31: 93-108. PMID: 23159179.

[2] Blaumeiser, B., van der Goot, I., Fimmers, R., Hanneken, S., Ritzmann, S., Seymons, K., Betz, R. C., Ruzicka, T., Wienker, T. F., De Weert, J., Lambert, J., Kruse, R., and Nothen, M. M. 2006. "Familial Aggregation of Alopecia Areata." J. Am. Acad. Dermatol. 54: 627-32. PMID: 16546583.

[3] Jagielska, D., Redler, S., Brockschmidt, F. F., Herold, C., Pasternack, S. M., Bartels, G. N., Hanneken, S., Eigelshoven, S., Refke, M., Barth, S., Giehl, K. A., Kruse, R., Lutz, G., Wolff, H., Blaumeiser, B., Bohm, M., Blume-Peytavi, U., Becker, T., Nothen, M. M., and Betz, R. C. 2012. "Follow-up Study of the First Genome-Wide Association Scan in Alopecia Areata: IL13 and KIAA0350 as Susceptibility Loci Supported with Genome-Wide Significance.” J. Invest. Dermatol. 132: 2192-7. PMID: 22534877.
[4] Messenger, A. G., McKillop, J., Farrant, P., McDonagh, A. J., and Sladden, M. 2012. "British Association of Dermatologists' Guidelines for the Management of Alopecia Areata." Br. J. Dermatol. 166: 916-26. PMID: 22524397.

[5] Bertolini, M., Gilhar, A., and Paus, R. 2012. "Alopecia Areata as a Model for T Cell-Dependent Autoimmune Diseases.” Exp. Dermatol. 21: 477-9. PMID: 22621196.

[6] Delogu, L. G., Deidda, S., Delitala, G., and Manetti, R. 2011. "Infectious Diseases and Autoimmunity." J. Infect Dev. Ctries. 5: 679-87. PMID: 21997935.

[7] Shelton, B. G., Kirkland, K. H., Flanders, W. D., and Morris, G. K. 2002. "Profiles of Airborne Fungi in Buildings and Outdoor Environments in the United States." Appl. Environ. Microbiol. 68: 1743-53. PMID: 11916692.

[8] Rudnicka, L., and Lukomska, M. 2012. “Alternaria Scalp Infection in a Patient with Alopecia Areata. Coexistence or Causative Relationship?” J. Dermato. Case report.

[9] Ellis, D., Davis, S., Alexiou, H., Handke, R., and Bartley, R. 2007. "Description of Medical Fungi." 2nd ed. Mycology Unit. Women's and children's hospital, North Adelaide, Australia.

[10] De-Hoog, G. S., Cuarro, J., Gene, J., and Figueras, M. J. 2000. "Atlas of Clinical Fungi." 2nd ed. Schimmelcultures, Utrecht, the Netherlands \& Universitate Rovira; Virgili, Reus, Spain.

[11] Hedayati, M. T., Arabzadehmoghadam, A., and Hajheydari, Z. 2009. "Specific IgE against Alternaria Alternata in Atopic Dermatitis and Asthma Patients." Eur. Rev. Med. Pharmacol. Sci. 13: 187-91. PMID: 19673169.

[12] Naidu, J. 1993. "Growing Incidence of Cutaneous and Ungual Infections by Non-Dermatophyte Fungi at Jabalpur (M.P.).” Indian J. Pathol. Microbiol. 36: 113-8. PMID: 8276472.

[13] Sun, J., Silva, K. A., McElwee, K. J., King, L. E. J., and Sundberg, J. P. 2008. "The C3H/HeJ Mouse and DEBR rat Models for Alopecia Areata: Review of Preclinical Drug Screening Approaches and Results.” Exp. Dermatol. 17: 793-805. PMID: 18798913.

[14] Revankar, S. G., and Sutton, D. A. 2010. "Melanized Fungi in Human Disease." Clin. Microbiol. Rev. 23: 884-928. PMID: 20930077.

[15] Plonka, P. M., and Grabacka, M. 2006. "Melanin Synthesis in Microorganisms-Biotechnological and Medical Aspects." Acta. Biochim. Pol. 53: 429-43. PMID: 16951740.

[16] Dorđić, M., Matić, I. Z., Filipović-Lješković, I., Džodić, R., Sašić, M., Erić-Nikolić, A., Vuletić, A., Kolundžija, B., Damjanović, A., Grozdanić, N., Nikolić, S., Pralica, J., Dobrosavljević, D., Rašković, S., Andrejević, S., and Juranić, Z. 2012. "Immunity to Melanin and to Tyrosinase 
in Melanoma Patients, and in People with Vitiligo.” $B M C$ 12: 109 .

[17] Kobayashi, T., Iijima, K., Radhakrishnan, S., Mehta, V., Vassallo, R., Lawrence, C. B., Cyong, J. C., Pease, L. R., Oguchi, K., and Kita, H. 2009. "Asthma-Related Environmental Fungus, Alternaria, Activates Dendritic Cells and Produces Potent Th2 Adjuvant Activity." J. Immunol. 182: 2502-10. PMID: 19201906.

[18] Zhao, Y., Zhang, B., Caulloo, S., Chen, X., Li, Y., and Zhang, X. 2012. "Diffuse Alopecia Areata Is Associated with Intense Inflammatory Infiltration and CD8+ T Cells in Hair Loss Regions and an Increase in Serum IgE Level." Indian J. Dermatol Venereol Leprol 78: 709-14. PMID: 23075639.

[19] Barahmani, N., Lopez, A., Babu, D., Hernandez, M., Donley, S. E., and Duvic, M. 2010. "Serum T Helper 1 Cytokine Levels Are Greater in Patients with Alopecia
Areata Regardless of Severity or Atopy." Clin. Exp. Dermatol 35: 409-16. PMID: 19874320.

[20] Teraki, Y., Imanishi, K., and Shiohara, T. 1996. "Cytokines in Alopecia Areata: Contrasting Cytokine Profiles in Localized Form and Extensive Form (Alopecia Universalis)." Acta Derm Venereol. 76: 421-23. PMID: 8982401.

[21] Knutsen, A. P., Bush, R. K., Demain, J. G., Denning, D. W., Dixit, A., Fairs, A., Greenberger, P. A., Kariuki, B., Kita, H., Kurup, V. P., Moss, R. B., Niven, R. M., Pashley, C. H., Slavin, R. G., Vijay, H. M., and Wardlaw, A. J. 2012. "Fungi and Allergic Lower Respiratory Tract Diseases." J. Allergy Clin. Immunol 129: 280-91; quiz 292-3. PMID: 22284927.

[22] Pastor, F. J., and Guarro, J. 2008. "Alternaria Infections: Laboratory Diagnosis and Relevant Clinical Features." Clin. Microbiol. Infect. 14: 734-46. PMID: 18727797. 